\title{
On the economic case for deinstitutionalisation in post-communist Europe
}

\author{
Christopher J Gerry ${ }^{1,2}$
}

\author{
${ }^{1}$ Oxford School of Global and Area Studies, University of Oxford, England. Phone: +44 1865 \\ 312697. Email: christopher.gerry@sant.ox.ac.uk and ${ }^{2}$ Centre for Health Economics, Management, \\ and Policy, National Research University Higher School of Economics, St. Petersburg, Russian \\ Federation.
}

Across the European region, human rights and clinical practice arguments have driven the contemporary model of mental health care, integrating community-based and primary care provision with shorterterm inpatient treatment, medication, talk therapies, social support and rehabilitative services ${ }^{1}$. Deinstitutionalisation, involving the discharge of psychiatric patients from large institutions into community-based care settings, has been at the heart of this development. The World Health Organization's European Mental Health Action Plan captures the consensus that "care and treatment should be provided in local settings, since large mental hospitals often lead to neglect and institutionalisation" ${ }^{2}$. However, many European countries, particularly those in the post-communist world, of Central and Eastern Europe and the Former Soviet Union, remain a long way from achieving these best-practice models, even when their legislative commitments apparently bind them.

The post-communist region represents a particularly interesting case since, on the one hand, traditions of psychiatric care are predominantly of the 'in-hospital' variety, and on the other hand, the postcommunist transition resulted in social, economic and political changes which placed an unusual set of pressures on the mental well-being of the population, while simultaneously exacerbating the shortage of resources necessary to deal with changing population health needs. Following intense international scrutiny, almost three decades later, the countries of this region have progressed with the modernisation of their mental health care provision. The initial reformulation of policies was informed by WHO principles requiring governments to initiate and consolidate the transition from institutionalised care towards community-oriented services ${ }^{3}$. New legislative statements ushered in enhanced patient rights and controls on hospitalisation; paved the way for a multi-disciplinary and multi-sectoral approach, involving social workers, psychologists and psychotherapists; and gave rise to a role for civil society in the provision and monitoring of psychiatric care. More recent reforms have focused specifically on the demands for deinstitutionalisation.

While improvements have been made and progressive official policy positions have been adopted, in practice, the mental health care systems of these countries remain principally based on institutional care. A gap exists and persists between the principles of deinstitutionalisation declared formally in the policy frameworks and the day-to-day manner in which mental health care is implemented 'on the ground'. Large, outdated, long- and repeat-stay psychiatric hospitals remain the main beneficiaries of the mental health care budgets and the conduits through which much of the care continues to be channelled ${ }^{4}$. This draws attention to the low priority assigned to mental health care by the major stakeholders and to the shortfall of resources necessary to finance reforms.

In this context, Winkler et al.'s prospective study on the cost-effectiveness of treating psychiatric patients in the community rather than with hospital-based care in the Czech Republic breaks new ground $^{5}$. While economic evidence, gathered in evaluation studies, has been used in Western Europe to argue the cost effectiveness of community-based care ${ }^{6}$, no equivalent evidence has been made available to policy makers in the post-communist region. Despite the myriad obstacles to collecting the data required to weigh the evidence of both the costs and benefits associated with complex interventions in mental health, Winkler et al. have taken a crucial first step towards addressing these challenges by cataloguing a systematic and pragmatic approach to economic evaluation in this setting. 
Aside from the studies undoubted novelty, this work is particularly important for at least two reasons. First, in a typical post-communist context, it provides evidence that the reallocation of resources towards community care settings and away from the long-term hospitalisation of psychiatric patients is associated with economic gains. This type of evidence can provide fiscal impetus for accelerating the reform process and can powerfully augment those arguments from human rights and clinical practice that may have less immediate traction with policy makers in post-communist countries. Second, there is very little health economic evaluation at all in the post-communist region and none in the mental health field. In providing a well-designed and carefully conducted blueprint for evaluation in this setting, the authors have invited others in the region to develop a more evaluative, evidence-based policy culture and to contribute to the relatively nascent sub-discipline of mental health economics.

Notwithstanding this, substantial obstacles lie in wait for those working in this area, particularly as concerns data availability and reliability in these post-communist settings. While the pragmatic decisions and assumptions made in this work are plausible and the limitations are transparently addressed, the challenges in the context of these countries remain daunting. To reliably calculate the full health and social costs and benefits; to form cooperative partnerships with relevant stakeholders; and to plausibly adapt existing methodologies to arrive at credible estimates of QALYs and costeffectiveness ratios, is difficult in the most accommodating of settings, but nowhere are the complexities and challenges greater than in the post-communist countries of Central and Eastern Europe and the Former Soviet Union.

However, if in these countries, the advance of community care is a crucial part of the policy mix required to "empower and emancipate people with psychiatric and social problems, enabling them to be fully participating members of society"7 then this study is an important marker in the development of an evidence base that can win over policy makers and stakeholders.

\section{References}

1. McCulloch A, Muijen M. Management issues in the mental health sector. In: Walshe K, Smith J. (eds.) Healthcare Management. Maidenhead: McGraw-Hill, Open University Press, 2011.

2. World Health Organization. European Mental Health Action Plan. 2013 http://www.euro.who.int/ data/assets/pdf file/0020/280604/WHO-Europe-Mental-HealthAcion-Plan-2013-2020.pdf (Accessed 20 October 2018).

3. Shek O, Pietilä I. The Limits for Deinstitutionalization of Psychiatry in Russia: Perspectives of Professionals Working in Outpatient Mental Health Services. International Journal of Mental Health 2016; 45: 118-134.

4. Petrea I. Mental health in former soviet countries: From past legacies to modern practices. Public Health Reviews 2013; 34(2).

5. Winkler P, Koeser L, Kondratova L, Broulikova H, Pav M, Kalisova L, Barrett B, McCrone P. An economic rationale for mental health care reform in the Czech Republic: cost-effectiveness of care for people with psychosis in the community and psychiatric hospitals. The Lancet Psychiatry 201X; (forthcoming).

6. Knapp M, Beecham J, McDaid D, Matosevic T, Smith M. The economic consequences of deinstitutionalisation of mental health services: lessons from a systematic review of European experience. Health and Social Care in the Community 2011; 19(2): 113-125.

7. Bauduin D, McCulloch A, Liegeois A. Good care in the community. Ethical aspects of deinstitutionalisation in mental health care. Utrecht: Trimbos instituut. 2002. 